\section{Caracterização gerencial dos hospitais filantrópicos no Brasil}

\author{
Management characteristics \\ in charity hospitals in Brazil
}

\author{
Sheyla Maria Lemos Lima ${ }^{1}$ \\ Pedro Ribeiro Barbosa 1 \\ Margareth C. Portela 1 \\ Maria Alicia Dominguez Ugá 1 \\ Miguel Murat Vasconcellos 1 \\ Silvia Gerschman 1
}

\footnotetext{
1 Departamento

de Administração e Planejamento em Saúde, Escola Nacional de Saúde Pública, Fundação Oswaldo Cruz, Rio de Janeiro, Brasil.

Correspondência

S. M. L. Lima

Departamento

de Administração

e Planejamento

em Saúde, Escola Nacional de Saúde Pública,

Fundação Oswaldo Cruz.

Rua Leopoldo Bulhões 1480,

Rio de Janeiro, $R J$

21041-210, Brasil.

slemos@ensp.fiocruz.br
}

\begin{abstract}
This paper presents the management characteristics of charity hospitals in Brazil, based on data from a national survey developed in 2001. The sample accounted for the random inclusion of 66 Brazilian Unified Health System (SUS) inpatient care providers with less than 599 beds and all 26 hospitals with at least 599 beds. It also included 10 institutions assumed as nonproviders of services to the SUS. The analyses are descriptive, focusing on the classification of the hospitals according to their managerial development level, as well as selected issues regarding the utilization of specific managerial technologies, human resources, technical services, and services contracting. Distinct managerial levels were identified, but it is important to note that 83\% of the SUS providers with less than 599 beds were classified as having incipient management. The authors discuss implications of the findings for inpatient care policies, considering the importance of charity hospitals for the Brazilian Health System.
\end{abstract}

Hospitals; Voluntary Hospitals; Hospital Administration; Management

\section{Introdução}

O setor hospitalar filantrópico no Brasil é atualmente responsável por cerca de 1/3 dos leitos existentes no país (http://www.datasus.gov. br). Caracteriza-se como importante prestador de serviços ao Sistema Único de Saúde (SUS), assim como à saúde suplementar. Requer ser mais bem conhecido, tendo em vista a formulação mais ajustada de políticas governamentais, objetivando a sua manutenção, desenvolvimento e maior integração ao sistema de saúde brasileiro, nas esferas pública e privada.

Pela regulamentação vigente, são consideradas filantrópicas as entidades portadoras do Certificado de Entidade Beneficente de Assistência Social (CEAS), concedido pelo Conselho Nacional de Assistência Social (CNAS), órgão subordinado ao Ministério de Assistência e Promoção Social. O CEAS é uma exigência para a obtenção de diversas isenções fiscais e tributárias, passíveis de serem auferidas pelas filantrópicas. Várias condições são exigidas para a concessão do CEAS. Em particular, para os hospitais, destacam-se duas exigências alternativas: a oferta e efetiva prestação de $60 \%$ ou mais de internações ao SUS; e, no caso do gestor local do SUS declarar impossibilidade de contratação de $60 \%$ de internações, a entidade hospitalar aplicar um porcentual da receita bruta em gratuidade, variando entre $20 \%$ e $5 \%$, na dependência do efetivo porcentual de atendimento ao SUS 1 . 
Este artigo apresenta parte dos resultados do Estudo sobre os Hospitais Filantrópicos no Brasil, que objetivou caracterizar a rede hospitalar filantrópica no país segundo as dimensões institucional, assistencial, gerencial e econômico-financeira, bem como quanto a relações com o mercado de saúde suplementar e com a rede pública do SUS. A dimensão gerencial constitui-se no foco deste artigo.

\section{Considerações conceituais}

A caracterização apresentada centra-se em variáveis de estruturas gerenciais, calcando-se no racional de que essas estruturas, refletindo o nível de organização formal e a capacidade gerencial, afetam o desempenho e sustentabilidade de serviços e sistemas de saúde 2 . Assume-se a premissa de que uma estrutura organizacional bem desenhada é condição necessária, ainda que não suficiente, para o bom desempenho de uma organização 3,4. Considerase, ainda, que as características organizacionais podem ser facilitadoras ou não de modelos de assistência 5 , estando esses modelos mais ou menos engajados na produção de cuidados de elevada qualidade, isto é, ajustados ao conhecimento profissional corrente e capazes de aumentar a probabilidade de ocorrência de resultados desejáveis 6 .

Por outro lado, a abordagem multidimensional utilizada está em conformidade com a tendência observada na literatura referente ao desempenho gerencial de organizações de saúde $7,8,9$, que contempla os oito processos de decisão apontados por Griffith 10, largamente considerados e adotados em sistemas de acreditação e premiação do setor, quais sejam: governança e gerenciamento estratégico; qualidade clínica; organização clínica; planejamento financeiro; planejamento e marketing; serviços de informação; recursos humanos; e planta física e suprimentos. $\mathrm{O}$ instrumento utilizado na caracterização gerencial apresentada, entretanto, diferencia-se na sua finalidade. Esta caracterização não considera indicadores de resultados em nenhuma das dimensões contempladas e tampouco permite avaliar a qualidade assistencial 11 . Tendo em vista as premissas já mencionadas e o entendimento de que o bom cuidado reflete adequação e eficiência de produção 12,13 , a idéia é proporcionar uma classificação de hospitais que tem como base suas estruturas gerenciais em diversas dimensões, sem avançar nas correlações entre tais estruturas e os respectivos desempenhos organizacionais, incluindo, em última análise, a qualidade assistencial. Esta é uma área que merece desenvolvimento, tendo em vista que ainda poucos trabalhos encontrados na literatura apontam nessa direção, sendo a maior parte estudos que analisam apenas o efeito de pressões financeiras e do credenciamento de corpo médico sobre a qualidade do cuidado em hospitais 14,15.

\section{Métodos}

Este estudo é transversal e baseia-se em dados coletados a partir da aplicação de um questionário em entrevista por um pesquisador.

Considerou-se, baseando-se no universo de entidades hospitalares filantrópicas no Brasil, três subconjuntos: (1) uma amostra aleatória de 66 entidades prestadoras de serviços ao SUS selecionadas em um universo de 1.658 com menos de 599 leitos - subconjunto designado como amostra; (2) as 27 entidades prestadoras de serviços ao SUS com pelo menos 599 leitos subconjunto designado como grandes entidades; e (3) dez entidades selecionadas intencionalmente, com base no reconhecimento do mercado de sua qualidade assistencial, no universo das filantrópicas não prestadoras de serviços ao SUS - subconjunto designado como hospitais especiais. No primeiro segmento foram registradas quatro perdas por razões diversas, repostas por hospitais de uma lista de reserva; as 66 entidades incluídas no estudo correspondem a 69 hospitais. No universo das grandes entidades, houve uma recusa sem reposição, resultando na inclusão de 15 hospitais individuais e 11 conglomerados - entidades constituídas por pelo menos dois hospitais -, envolvendo um total de 81 hospitais. No terceiro subconjunto houve três recusas com reposição.

O questionário envolveu questões fechadas e semi-abertas relativas a aspectos institucionais, assistenciais e gerenciais das entidades/ hospitais; ainda considerou elementos de suas relações com o mercado de saúde suplementar e com a rede pública do SUS.

No caso dos hospitais pertencentes a conglomerados, especificamente, o instrumento de coleta de dados foi preenchido pelos próprios hospitais, sendo o pesquisador de campo responsável somente pelo preenchimento do questionário da sede.

As análises aqui apresentadas são essencialmente descritivas.

Como ferramenta de análise das características gerenciais dos hospitais foi construído um sistema de classificação incluindo seis dimensões da estrutura de gestão hospitalar 10: direção e planejamento, econômico-financei- 
ra, recursos humanos, serviços técnicos, serviços logísticos e tecnologia de informações. Cada dimensão compreende um conjunto de variáveis tomadas como marcadoras do nível de desenvolvimento gerencial, tendo sido consideradas, no total, 82 variáveis (Tabelas 1, 2, 3, $4,5$ e 6$)$.

Segundo a classificação proposta, cada uma das dimensões é enquadrada em um nível de desenvolvimento - avançado, em desenvolvimento ou incipiente - a partir da presença ou ausência das variáveis marcadoras. Este processo é realizado a partir do nível avançado. Observa-se se todos os requisitos para a classificação da dimensão em avançado, tratada como benchmarking, são atendidos, isto é, se há conformidade em todas as variáveis utilizadas. No caso de não o serem, verifica-se o cumprimento dos requisitos exigidos para o nível $\mathrm{em}$ desenvolvimento. Enfim, classifica-se a dimensão como incipiente, caso não estejam presentes os requisitos exigidos na categoria em $\mathrm{de}$ senvolvimento.

A seguir, atribui-se um escore à dimensão, considerando o enquadramento no nível de desenvolvimento gerencial definido e a pontuação correspondente às condições adicionais presentes, estabelecidas como requisitos da categoria imediatamente superior.

Para os níveis incipiente e em desenvolvimento, que totalizam escores variando entre 0 e 4,9 pontos, e 5,0 e 9,9, respectivamente, esses

\begin{tabular}{|c|c|c|c|}
\hline Condição & Incipiente & Em desenvolvimento & Avançado \\
\hline \multicolumn{4}{|l|}{ 01. Dirigente executivo hospitalar - número de horas dedicadas à função } \\
\hline Pelo menos 40 horas & - & - & $\mathrm{X}$ \\
\hline Entre 20 e 39 horas & - & $X$ & - \\
\hline 02. Dirigente executivo hospitalar - curso superior completo & - & $X$ & $x$ \\
\hline 03. Dirigente executivo hospitalar - pós-graduação em gestão hospitalar ou similar & - & - & $\mathrm{x}$ \\
\hline \multicolumn{4}{|l|}{ 04. Cargos formalizados de Direção } \\
\hline 5 ou mais & - & - & $\mathrm{X}$ \\
\hline 2 a 4 & - & $\mathrm{X}$ & - \\
\hline 05. Organograma formal & - & $\mathrm{x}$ & $x$ \\
\hline 06. Plano formal periódico (ao menos anual) & - & - & $X$ \\
\hline 07. Metodologia de planejamento - mais de três aspectos contemplados & - & - & $X$ \\
\hline 08. Componentes do plano - mais de três componentes presentes & - & - & $X$ \\
\hline 09. Orçamento anual & - & $\mathrm{X}$ & $\mathrm{X}$ \\
\hline 10. Marketing - canal de comunicação com a clientela & - & $\mathrm{X}$ & $\mathrm{X}$ \\
\hline $\begin{array}{l}\text { 11. Marketing - oferta de serviços a partir da identificação de necessidades } \\
\text { de mercado/clientes ou propaganda de produtos/serviços oferecidos }\end{array}$ & - & - & $\mathrm{X}$ \\
\hline 12. Avaliação de desvio orçamentário & - & - & $\mathrm{X}$ \\
\hline \multicolumn{4}{|l|}{$\begin{array}{l}\text { 13. Avaliação com metas e indicadores específicos para os serviços assistenciais, } \\
\text { administrativos e logísticos }\end{array}$} \\
\hline Sistema global integrado & - & - & $\mathrm{X}$ \\
\hline Somente sistemas em serviços isolados & - & $\mathrm{X}$ & - \\
\hline
\end{tabular}

Metodologia de planejamento - aspectos contemplados: (1) avaliação de mercado; (2) políticas/tendências do SUS;

(3) avaliação de pontos fortes e fracos internos e externos; (4) definição da missão da organização; (5) construção de cenários; (6) definição de ações/implementação de projetos; (7) estabelecimento de metas e prazos; (8) permanente monitoramento de metas e prazos; (9) balanço dos objetivos/metas de planos anteriores; (10) ajustes periódicos. Componentes do plano: (1) projeto assistencial tratando de abertura de serviços e/ou metas assistenciais:

(2) programação de atividades assistenciais com metas estabelecidas para (a) consultas ambulatoriais, (b) atendimento na emergência, (c) internações e (d) cirurgias; (3) associação/parceria com outras organizações; (4) investimento tecnológico - aquisição, reposição de equipamentos; (5) obras e/ou reformas; (6) desenvolvimento de recursos humanos - formação e treinamento; (7) política de mercado - crescimento e/ou novas áreas de atuação; (8) política de financiamento/endividamento; (9) modernização gerencial - incluindo novos sistemas, treinamento gerencial; (10) desenvolvimento de tecnologias de informação; (11) abertura de novos negócios - hospitalares e não hospitalares;

(12) orçamento com base nas atividades programadas; (13) balanço das metas alcançadas - no caso de haver plano anterior. Sistema Global Integrado: sistema abrangente, coordenado na direção do hospital, articulando subsistemas de avaliação em serviços administrativos (administração central e demais serviços logísticos gerais) e assistenciais (setores de internação, ambulatórios, emergência, home care e serviços de diagnóstico e terapia).

Somente sistemas em serviços isolados: apenas alguns serviços administrativos ou assistenciais possuem sistemas próprios não estando coordenados de forma articulada na direção do hospital. 
Estrutura econômico-financeira.

\begin{tabular}{|c|c|c|c|}
\hline Condição & Incipiente & Em desenvolvimento & Avançado \\
\hline 01. Diretor/chefe financeiro - cargo formal ou informal & - & $x$ & $x$ \\
\hline $\begin{array}{l}\text { 02. Diretor/chefe financeiro - curso superior completo na área econômico-financeira } \\
\text { ou pós-graduação na área econômico-financeira (para graduações em outras áreas) }\end{array}$ & - & - & $\mathrm{x}$ \\
\hline 03. Balanço fechado* & - & - & $x$ \\
\hline 04. Balancete até 60 dias & - & - & $\mathrm{x}$ \\
\hline 05. Auditoria contábil independente & - & - & $x$ \\
\hline 06. Auditor cadastrado na Comissão de Valores Mobiliários & - & - & $x$ \\
\hline 07. Balancete periódico de patrimônio e resultados & - & - & $x$ \\
\hline 08. Controle financeiro de estoque & - & $x$ & $x$ \\
\hline $\begin{array}{l}\text { 09. Inventário, pelo menos anual, dos materiais hospitalares (permanentes, } \\
\text { medicamentos, material de consumo e imobilizados - equipamentos e viaturas) }\end{array}$ & - & $x$ & $x$ \\
\hline 10. Regras de depreciação patrimonial & - & - & $x$ \\
\hline 11. Projeção de fluxo de caixa & - & $x$ & $x$ \\
\hline 12. Seguro dos principais ativos - pelo menos três dos listados & - & - & $x$ \\
\hline 13. Investimentos financeiros & - & - & $x$ \\
\hline 14. Plano próprio de contas & - & $x$ & $x$ \\
\hline \multicolumn{4}{|l|}{ 15. Controle de contas } \\
\hline Informatizado & - & - & $x$ \\
\hline Manual & - & $x$ & - \\
\hline 16. Despesa conhecida por paciente ou procedimento & - & - & $x$ \\
\hline
\end{tabular}

* Para respostas obtidas até 31 de março, considerar balanço do ano anterior ao anterior; para respostas obtidas

a partir de 1o de abril, considerar balanço do ano anterior.

Seguros considerados: (1) equipamentos; (2) prédios; (3) estoque; (4) caixa; (5) valores em trânsito; (6) veículos; (7) outros.

são atribuídos em base proporcional ao número de quesitos "extras" (isto é, que se adicionam aos requisitos mínimos da categoria em que se enquadra o hospital) verificados. O nível avançado corresponde a dez pontos, expressando conformidade com todas as exigências.

Pretendeu-se diferenciar hospitais que, embora pertencendo a uma mesma categoria, apresentassem condições gerenciais diferentes, mas teve-se o cuidado de impedir que os hospitais que cumprissem vários requisitos inerentes à categoria superior (mas não todos) subissem de categoria.

Assim, por exemplo, considerando a dimensão recursos humanos, um hospital classificado na categoria em desenvolvimento (cuja "nota" básica é 5) que exibisse 2 dos 11 requisitos da categoria avançado, não exigidos na categoria em desenvolvimento, receberia 5,91 pontos na dimensão, correspondentes à expressão aritmética: $5+2 x(5 / 11)$.

O escore final de cada hospital é a média ponderada dos escores por ele obtidos em cada uma das seis dimensões, considerando os seguintes pesos: peso 2 para direção e instrumentos de planejamento, econômico-financeira e recursos humanos; peso 1,5 para logística e serviços técnicos; e peso 1 para tecnologia de informações.

O conjunto de variáveis utilizadas nesta tipologia não foi inteiramente coletado junto aos hospitais componentes dos conglomerados, impedindo assim a aplicação da mesma naquele conjunto de hospitais. De igual maneira não é aplicável a entidades filantrópicas constituídas como fundações de apoio, uma vez que estas não apresentam características típicas comuns aos sistemas de gestão hospitalar, objeto de referência desta tipologia. Dessa forma, as análises considerando a classificação gerencial envolveram somente: 63 hospitais da amostra (dos 69 hospitais foram excluídos uma fundação de apoio e cinco hospitais componentes de dois conglomerados); 13 grandes hospitais individuais (das grandes entidades foram excluídos os conglomerados e dois hospitais individuais que correspondiam a fundações de apoio) e os dez hospitais especiais.

Para o conjunto de todos os hospitais do estudo foi possível, entretanto, analisar a condição gerencial com base em alguns instrumentos de gestão, integrantes ou não do sistema de classificação. 
Estrutura de gestão de recursos humanos.

\begin{tabular}{|c|c|c|c|}
\hline Condição & Incipiente & Em desenvolvimento & Avançado \\
\hline 01. Diretor/chefe de RH - cargo formal ou informal & - & $x$ & $x$ \\
\hline 02. Diretor/chefe de RH - profissional de nível superior & - & - & $x$ \\
\hline \multicolumn{4}{|l|}{ 03. Incentivos de RH } \\
\hline 4 ou mais incentivos presentes & - & - & $x$ \\
\hline Até 3 incentivos presentes & - & $x$ & - \\
\hline \multicolumn{4}{|l|}{ 04. Treinamento técnico de RH da área assistencial - excluídos médicos } \\
\hline Mais de 25\% dos funcionários da área assistencial & - & - & $x$ \\
\hline Entre 5 e $25 \%$ dos funcionários da área assistencial & - & $x$ & - \\
\hline \multicolumn{4}{|l|}{ 05. Treinamento técnico de RH da área administrativa - excluídos gerentes } \\
\hline Mais de $10 \%$ dos funcionários da área administrativa & - & - & $x$ \\
\hline Existência de treinamento, incluindo até $10 \%$ dos funcionários da área administrativa & - & $x$ & - \\
\hline 06. Treinamento gerencial & - & - & $x$ \\
\hline 07. Plano de cargos & - & - & $x$ \\
\hline 08. Tabela salarial estruturada & - & - & $x$ \\
\hline 09. Avaliação sistemática de RH & - & - & $x$ \\
\hline 10. Existência de normas de funcionamento do corpo clínico & - & - & $x$ \\
\hline \multicolumn{4}{|l|}{ 11. Rotatividade de pessoal CLT } \\
\hline Até $10 \%$ & - & - & $x$ \\
\hline Entre 10 e $30 \%$ & - & $x$ & - \\
\hline $\begin{array}{l}\text { 12. Recrutamento de pessoal predominantemente feito por meio } \\
\text { de seleção pública ou de currículos }\end{array}$ & - & - & $x$ \\
\hline
\end{tabular}

Incentivos considerados: (1) ganhos adicionais e variáveis associados à produção e qualidade - alcance de metas: (2) pecúnios/prêmios por antigüidade/tempo de serviço no hospital; (3) pecúnios/prêmios por assiduidade; (4) pecúnios/ prêmios por inovação; (5) participação em congressos; (6) apoio a pesquisas; (7) apoio a linhas de formação - cursos, estágios etc.; (8) aquisição de publicações científicas; (9) cobertura assistencial com planos de saúde para funcionários e dependentes; (10) planos de previdência privada; (11) capacitação técnico-profissional; (12) capacitação gerencial. Rotatividade de pessoal 20: Admitidos + Saídos $\times 100$

\section{Resultados}

\section{Classificação gerencial}

Entre os 63 hospitais da amostra, a grande maioria (83\%) foi considerada como de nível incipiente de gestão. Entre os 13 grandes hospitais individuais e dez especiais, a situação foi inversa, sendo a maioria classificada como em desenvolvimento (respectivamente, $62 \%$ e $90 \%$ ). Nenhum dos hospitais foi classificado como avançado.

As médias dos escores globais obtidas por cada um dos segmentos reiteram os resultados acima citados. Os hospitais da amostra apresentam uma média baixa, 3,4, e os hospitais individuais de grande porte e hospitais especiais apresentam médias mais elevadas, respectivamente, 6,1 e 7,3 (Figura 1). No conjunto dos hospitais pesquisados, nos três segmentos, também foi possível identificar as dimensões que mais se diferenciam em termos de desenvolvimento, refletindo maior conformidade aos requisitos contemplados.
Tomando isoladamente o segmento da amostra, as dimensões que obtiveram maior e menor média foram, respectivamente, serviços técnicos (4,6 pontos) e econômico-financeira (2,6 pontos) (Figura 1). Tais médias denotam o caráter incipiente de gestão dominante nesses hospitais, que são predominantemente pequenos, com limitada complexidade assistencial e porte financeiro.

No cruzamento da classificação gerencial com nível de complexidade assistencial, porte de leitos e porte financeiro, observa-se que dos hospitais da amostra que apresentam um desenvolvimento gerencial incipiente (83\%), 98\% são de baixa e média complexidade, $85 \%$ possuem menos de 100 leitos e $88 \%$ são de micro ou pequeno porte financeiro. Os $17 \%$ restantes, classificados como em desenvolvimento, apresentam-se com um perfil diferente do segmento majoritário da amostra. Desses, 91\% são de média e alta complexidade e $55 \%$ possuem mais de 100 leitos. Como no restante da amostra, prevalecem hospitais de micro e pequeno porte financeiro (64\%). 
Estrutura logística.

\begin{tabular}{|c|c|c|c|}
\hline Condição & Incipiente & Em desenvolvimento & Avançado \\
\hline 01. Chefe/Diretor de serviço de materiais - cargo formal ou informal & - & $x$ & $x$ \\
\hline 02. Chefe/Diretor de serviço de materiais com nível superior & - & - & $x$ \\
\hline 03. Compras centralizadas no hospital ou entidade vinculada & - & $x$ & $x$ \\
\hline 04. Cadastro de fornecedores & - & - & $x$ \\
\hline 05. Estoque - curva $A B C$ & - & - & $x$ \\
\hline 06. Material médico-cirúrgico padronizado & - & - & $x$ \\
\hline \multicolumn{4}{|l|}{ 07. Equipamentos patrimoniados } \\
\hline $60 \%$ ou mais & - & - & $x$ \\
\hline Entre 20 e $60 \%$ & - & $x$ & - \\
\hline \multicolumn{4}{|l|}{ 08. Manutenção } \\
\hline Predial, equipamentos biomédicos e outros & - & - & $x$ \\
\hline Equipamentos biomédicos e outros & - & $x$ & - \\
\hline \multicolumn{4}{|l|}{ 09. Equipe de manutenção - composição } \\
\hline Engenheiro clínico, civil, mecânico ou eletricista/eletrônico & - & - & $\mathrm{x}$ \\
\hline Técnico de equipamentos biomédicos, técnico de eletricidade ou técnico em mecânica & - & $\mathrm{x}$ & - \\
\hline \multicolumn{4}{|l|}{ 10. Manutenção preventiva de equipamentos biomédicos } \\
\hline $40 \%$ ou mais & - & - & $x$ \\
\hline Entre 20 e $40 \%$ & - & $x$ & - \\
\hline \multicolumn{4}{|l|}{ 11. Manuais de equipamentos biomédicos disponíveis } \\
\hline $40 \%$ ou mais & - & - & $x$ \\
\hline Entre 20 e $40 \%$ & - & $\mathrm{x}$ & - \\
\hline 12. Responsável pelo setor de limpeza com nível superior & - & - & $x$ \\
\hline $\begin{array}{l}\text { 13. Treinamento regular do pessoal de limpeza segundo normas de controle } \\
\text { da infecção hospitalar }\end{array}$ & - & $x$ & $x$ \\
\hline 14. Análise de água a cada seis meses & - & $\mathrm{x}$ & $x$ \\
\hline 15. Limpeza de reservatórios de água a cada seis meses & - & $\mathrm{x}$ & $x$ \\
\hline 16. Perfuro-cortantes separados de quaisquer outros resíduos & - & $x$ & $x$ \\
\hline $\begin{array}{l}\text { 17. Treinamento regular do pessoal da lavanderia segundo normas de controle } \\
\text { da infecção hospitalar }\end{array}$ & - & - & $x$ \\
\hline $\begin{array}{l}\text { 18. Treinamento regular do pessoal da recepção/zeladoria/portaria } \\
\text { sobre acolhimento/atendimento ao público }\end{array}$ & - & - & $x$ \\
\hline 19. Instalações elétricas conforme normas ABNT & - & - & $x$ \\
\hline $\begin{array}{l}\text { 20. Sistema de energia de emergência/gerador para áreas críticas (emergência, } \\
\text { UTI, centro cirúrgico) }\end{array}$ & - & $x$ & $x$ \\
\hline
\end{tabular}

Nos segmentos grandes hospitais individuais e hospitais especiais, as dimensões melhor e pior pontuadas foram tecnologia de informações e recursos humanos, respectivamente (Figura 1). Tecnologia de informações nos grandes hospitais individuais obteve a média 7,2 e nos especiais 8,6; recursos humanos nos grandes individuais obteve a média 4,8 e nos especiais, 6,7. A condição de hospitais de maior porte, com complexidades assistenciais bem diferenciadas e importantes valores de faturamento, acompanham-se, como esperado, de melhores estruturas de gestão, sem que no entanto, sejam minimizadas as necessidades de desenvolvimento, nas diversas dimensões:
- $\quad$ os grandes hospitais individuais (13) estão classificados, na sua maior parte (8), como em desenvolvimento; desses, sete são de média e alta complexidade, e um especializado, seis possuem mais de 600 leitos, assim como seis são de grande porte financeiro; cinco hospitais deste segmento são incipientes, dos quais três de baixa e média complexidade, e dois especializados; dos incipientes, quatro possuem mais de 301 leitos e quatro são de médio porte financeiro.

- dos hospitais especiais (10), nove estão classificados como em desenvolvimento, sendo seis de alta complexidade, cinco com 151 a 600 leitos, e seis de grande porte financeiro; o único hospital incipiente é de média complexidade, tem menos 
Estrutura dos serviços técnicos.

\begin{tabular}{|c|c|c|c|}
\hline Condição & Incipiente & Em desenvolvimento & Avançado \\
\hline \multicolumn{4}{|l|}{ 01. Laboratório de Análises clínicas próprio ou terceirizado } \\
\hline Capacidade de resposta por 24 horas/dia & - & - & $\mathrm{x}$ \\
\hline Capacidade de resposta inferior a 24 horas/dia & - & $x$ & - \\
\hline $\begin{array}{l}\text { 02. Responsável pelo Laboratório - profissional de nível superior - } \\
\text { bioquímico, biólogo, médico ou biomédico }\end{array}$ & - & $x$ & $\mathrm{x}$ \\
\hline 03. Laboratório com registro na Vigilância Sanitária & - & $x$ & $\mathrm{x}$ \\
\hline 04. Laboratório com certificado ASPAC ou SBPC & - & $x$ & $x$ \\
\hline \multicolumn{4}{|l|}{ 05. Serviço de Radiologia ou Imagem próprio ou terceirizado } \\
\hline Capacidade de resposta por 24 horas/dia & - & - & $x$ \\
\hline Capacidade de resposta inferior a 24 horas/dia & - & $x$ & - \\
\hline \multicolumn{4}{|l|}{ 06. Responsável pelo serviço de radiologia ou imagem } \\
\hline Médico especialista em imagem & - & - & $x$ \\
\hline Médico de outra especialidade & - & $x$ & - \\
\hline 07. Serviço de radiologia ou imagem com registro na Vigilância Sanitária & - & $x$ & $x$ \\
\hline $\begin{array}{l}\text { 08. Serviço de radiologia ou imagem com controle permanente de exposição } \\
\text { à radiação com dosímetro individual }\end{array}$ & - & - & $x$ \\
\hline \multicolumn{4}{|l|}{ 09. Farmacêutico } \\
\hline 40 horas & - & - & $\mathrm{x}$ \\
\hline Menos de 40 horas & - & $\mathrm{x}$ & - \\
\hline 10. Comissão de padronização de medicamentos & - & - & $\mathrm{x}$ \\
\hline 11. Dose unitária por paciente ou dose individual paciente-dia & - & - & $x$ \\
\hline \multicolumn{4}{|l|}{ 12. Prontuário dos pacientes } \\
\hline Único - para pacientes externos e internos & - & - & $x$ \\
\hline Separado - para pacientes externos e internos & - & $x$ & - \\
\hline \multicolumn{4}{|l|}{ 13. Serviço de nutrição próprio ou terceirizado } \\
\hline Atuação na produção alimentar e atenção nutricional & - & - & $\mathrm{x}$ \\
\hline Atuação somente na produção alimentar & - & $x$ & - \\
\hline
\end{tabular}

Estrutura em tecnologia de informações.

\begin{tabular}{|c|c|c|c|}
\hline Condição & Incipiente & Em desenvolvimento & Avançado \\
\hline 01. Serviço/núcleo/equipe de informática próprio ou terceirizado & - & $x$ & $x$ \\
\hline 02. Computadores interligados em rede interna & - & $x$ & $x$ \\
\hline 03. Ao menos um servidor & - & $x$ & $x$ \\
\hline 04. Sistema gerenciador de banco de dados & - & - & $x$ \\
\hline \multicolumn{4}{|l|}{ 05. Sistemas de informação automatizados } \\
\hline 4 ou mais programas existentes & - & - & $x$ \\
\hline até 3 programas & - & $x$ & - \\
\hline \multicolumn{4}{|l|}{ 06. Acesso à Internet } \\
\hline Permanente & - & - & $x$ \\
\hline Discado & - & $\mathrm{x}$ & - \\
\hline 07. Processo de contingência formalmente normatizado & - & - & $x$ \\
\hline $\begin{array}{l}\text { 08. Oferta de serviços pela Internet - inclui informações específicas para pacientes (marcação } \\
\text { de consultas, acesso a resultados de exames etc.) ou acesso aos prontuários para os médicos }\end{array}$ & - & - & $x$ \\
\hline
\end{tabular}


de cinqüenta leitos e é de médio porte financeiro (perfil semelhante ao dos hospitais da amostra).

Somente 3\% dos hospitais da amostra obtiveram escore final acima de 7 , condição observada em $31 \%$ dos grandes hospitais individuais e 7 dos 10 hospitais especiais.

\section{Uso de instrumentos gerenciais}

Considerando todos os hospitais incluídos no estudo por segmentos - hospitais da amostra
(69), componentes de conglomerados (81), grandes individuais (15) e hospitais especiais (10) -, observa-se uma crescente utilização de instrumentos gerenciais, expressando um gradiente de desenvolvimento gerencial (Figura 2).

Há uma menor diversidade e intensidade na utilização de instrumentos e práticas nos hospitais componentes dos conglomerados do que nos grandes hospitais individuais. Comportamento diferente quanto à intensidade é apresentado em relação à presença de Plano e

Figura 1

Hospitais filantrópicos segundo pontuações médias obtidas na classificação por dimensão. Brasil, 2001.

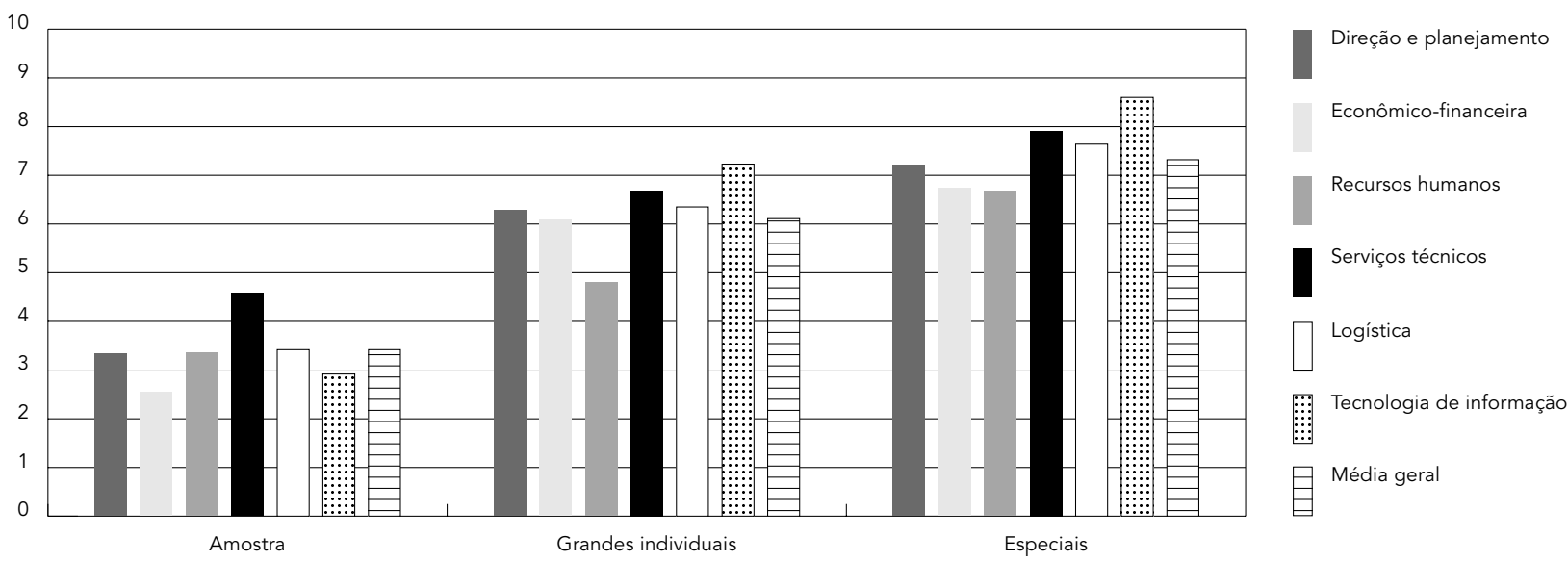

Figura 2

Hospitais filantrópicos segundo presença de alguns instrumentos de gestão. Brasil, 2001.

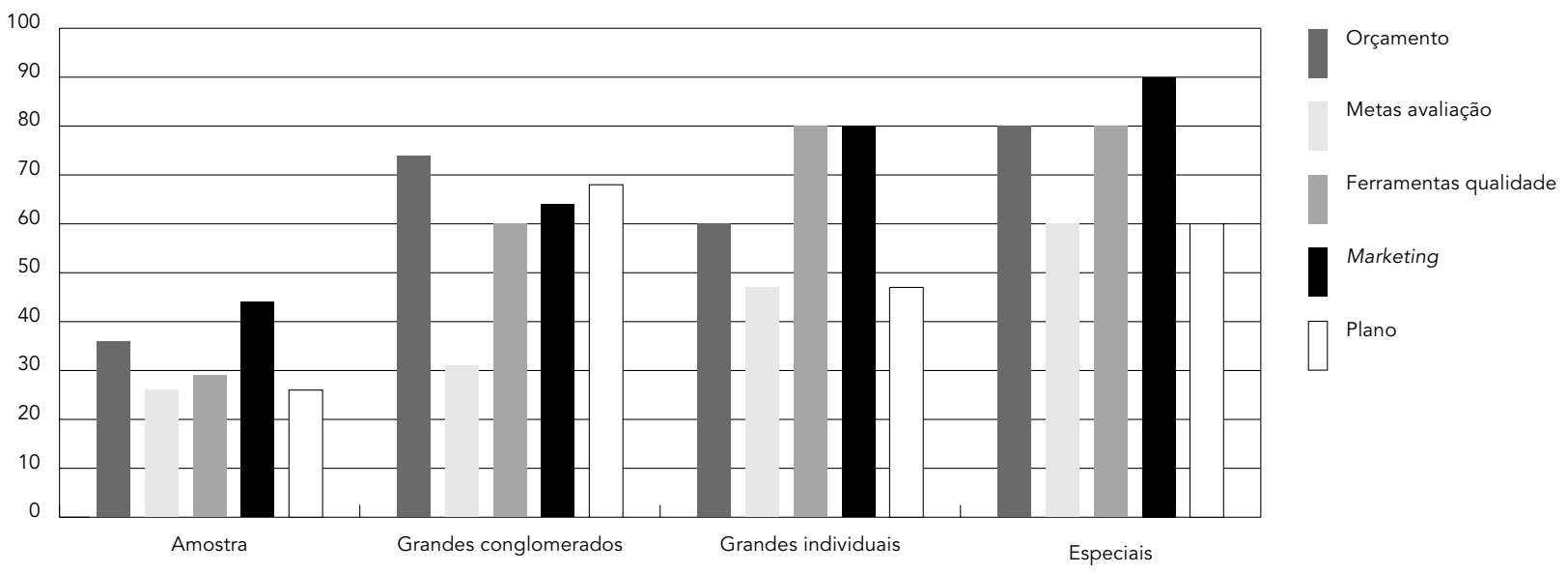


Orçamento formais, mais freqüentes nos hospitais conglomerados - respectivamente, em $68 \%$ e $74 \%$ dos hospitais - do que nos individuais - respectivamente, em $47 \%$ e $60 \%$ dos hospitais. Tal achado pode ser explicado pela necessidade desses instrumentos para a garantia de uma coordenação central por parte da entidade líder do conglomerado. Entretanto, como lógica predominante, essas entidades não apresentam racionalidades gerenciais típicas de complexos organizacionais, presentes em outros setores da economia. Somente 25 hospitais componentes $(31 \%)$ registram utilizar um sistema de avaliação de metas e indicadores, principal parâmetro de delineamento de uma estrutura conglomerada e de sua coordenação. No campo do planejamento hospitalar, importante discrepância entre componentes da mesma entidade denuncia a limitada coordenação do complexo hospitalar pela sede 16 .

A presença de sistema de avaliação nos demais segmentos é também precária; o segmento que mais refere a sua utilização é dos hospitais especiais -6 dos 10 hospitais.

Os grandes hospitais individuais $(80 \%)$ assim como os especiais (90\%) utilizam de forma mais enfática estratégias de marketing (canais de comunicação com os clientes e propaganda de serviços). Considerando somente os canais de comunicação com os clientes, os hospitais componentes dos conglomerados (64\%) e os da amostra (44\%) os utilizam de forma mais tímida.

A utilização de ferramentas de gerência da qualidade também evidencia a diferenciação entre os segmentos, estando presente em 9 dos 10 hospitais especiais, em $80 \%$ dos grandes hospitais individuais e em $60 \%$ dos componentes dos conglomerados. Na amostra há registro em somente $29 \%$ dos hospitais.

\section{Gestão de recursos humanos}

Em relação aos vínculos trabalhistas prevalentes, os profissionais médicos apresentam comportamento completamente distinto dos demais profissionais da área assistencial e não assistencial. Os médicos se relacionam com o hospital por meio de vínculos mais precários e instáveis, predominando a condição de cadastrado, em que estão autorizados a realizar atividades médicas como profissional autônomo, seja por intermédio de pessoa jurídica ou física. Exceção ocorre nos grandes hospitais individuais, cujo vínculo principal é celetista (28\%), seguido do vínculo como médico residente. Vale, entretanto, sublinhar que, mesmo no caso desses hospitais, os vínculos precários (cadastrados, terceirizados, autônomos e cooperati- vados) somados perfazem a maioria. O diferencial nos grandes hospitais individuais é, de fato, a presença de médicos residentes, que representavam, naquele segmento, $20 \%$ da força de trabalho médica, característica sempre importante em hospitais com atividades de ensino (Figura 3).

Do ponto de vista dos demais profissionais de nível superior assistencial e não assistencial, nível médio e elementar, a maioria é celetista. Destaca-se apenas a presença ainda significativa, na amostra, de profissionais de nível superior com vínculo terceirizado, autônomo ou cooperativado.

Ainda no campo dos recursos humanos, os hospitais apresentam níveis de rotatividade elevados. Exceto pela amostra, onde $40,6 \%$ dos hospitais apresentam rotatividade de até $10 \%$, prevalece nos segmentos de hospitais considerados uma rotatividade entre $10,1 \%$ e $20 \%$ ( $47 \%$ dos hospitais grandes individuais; $40 \%$ dos especiais e $33 \%$ dos hospitais componentes dos conglomerados). Com rotatividade superior a $40 \%$, registram-se $4 \%$ dos hospitais da amostra, $13 \%$ dos grandes hospitais individuais e $10 \%$ dos componentes dos conglomerados. Esses resultados merecem atenção, ainda que considerando: (1) a possibilidade de imprecisão de dados considerados no seu cálculo, face à dificuldade encontrada no levantamento dos quantitativos celetistas; (2) o fato deles referirem-se somente ao ano de 2000 , podendo re-

Figura 3

Hospitais filantrópicos segundo tipos de vínculos de profissionais médicos. Brasil, 2001.

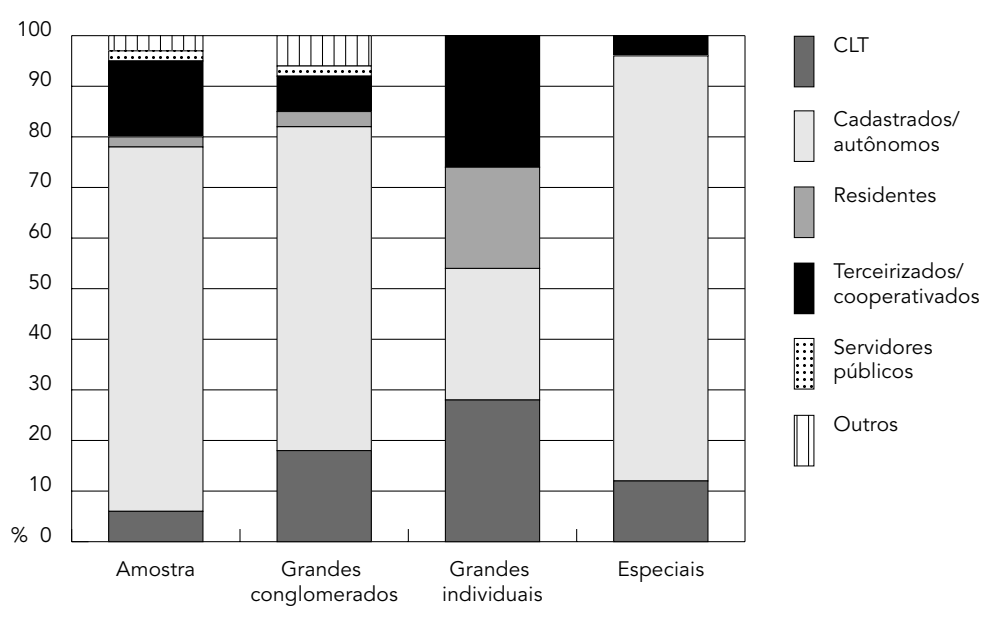


fletir, eventualmente, um comportamento atípico da organização; e (3) a ausência de um padrão para o nível de rotatividade aceitável no setor Saúde.

Os segmentos que mais referem a utilização de incentivos na gestão de $R H$ são os hospitais especiais e grandes individuais, sendo a capacitação gerencial o tipo de incentivo mais freqüente, presente em $73 \%$ dos grandes hospitais individuais e oito dos dez especiais. Tal incentivo está presente em somente $23 \%$ dos hospitais da amostra e $52 \%$ dos componentes dos conglomerados.

\section{Serviços técnicos}

Considerada a Farmácia, foram analisadas a forma de dispensação de medicamentos prevalente e a existência de comissão de padronização de medicamentos. A dispensação de medicamentos pode ser realizada de três diferentes maneiras, individualmente ou de forma associada. A dose individualizada - onde a Farmácia aloca os medicamentos do dia para o paciente, sem separação por horário de administração - e, especialmente, a dose unitária por paciente-dia - em que a Farmácia fraciona os medicamentos segundo as diversas administrações do dia para cada paciente - são consideradas boas práticas de assistência farmacêutica, assegurando maior racionalidade/menor desperdício na utilização de recursos e maior controle de qualidade da assistência em si. A terceira maneira, a dose coletiva, consiste na dispensação dos medicamentos sem nenhuma discriminação em relação às doses.

A presença de uma comissão de padronização atuante, por sua vez, indica maior racionalidade e melhor controle da qualidade da assistência pela reflexão permanente da utilização dos medicamentos frente às patologias atendidas e à disponibilização pelo mercado de novos produtos.

O tipo de dispensação mais observado foi a dose individualizada, valendo a ressalva que ela podia estar sendo utilizada com as outras formas de dispensação. Foi referida por 8 dos 10 hospitais especiais, $87 \%$ dos grandes individuais, $67 \%$ dos hospitais componentes dos conglomerados e $54 \%$ dos da amostra. Nos hospitais especiais não houve registro de utilização da dose coletiva.

Quanto à existência de comissão de padronização de medicamentos, foi referida por 7 dos 10 hospitais especiais, $67 \%$ dos grandes individuais, 59\% dos hospitais componentes de conglomerados e $35 \%$ dos da amostra.

No que se refere à organização do Serviço de Arquivo Médico e Estatístico (SAME), o pre- conizado é o prontuário único por paciente, que prevê o registro de sua história clínica, e procedimentos diagnósticos e terapêuticos utilizados, em qualquer atendimento (ambulatório, emergência, internação etc.) no hospital. Ele propicia a continuidade da assistência ao paciente, independentemente do serviço ou profissional que $o$ atende.

Neste aspecto, os diferentes segmentos são pouco diferenciados. O uso de prontuário único foi reportado por somente 3 dos 10 hospitais especiais, $20 \%$ dos grandes individuais, $28 \%$ dos da amostra e $28 \%$ dos hospitais componentes de conglomerados. A coexistência de prontuários separados para pacientes internos e externos foi registrada em 6 dos 10 hospitais especiais, $53 \%$ dos grandes hospitais individuais, $57 \%$ dos componentes de conglomerados e $51 \%$ dos da amostra. Com prontuário somente para o paciente interno, enumeram-se um hospital especial, 20\% dos grandes hospitais individuais, $22 \%$ dos componentes de conglomerados e $19 \%$ dos da amostra.

\section{Terceirização de serviços}

Supunha-se uma freqüência maior de terceirizações no interior dos hospitais. Em todos os segmentos, os serviços de diagnóstico e terapêutica (SADT) foram os mais citados como terceirizados. Entre eles, destacam-se: os serviços de patologia clínica e de radiologia, terceirizados, respectivamente, em $58 \%$ e $20,3 \%$ dos hospitais da amostra; os serviços de patologia clínica, ultra-sonografia, tomografia e hemodinâmica, terceirizados em $27 \%$ dos grandes hospitais individuais; os serviços de patologia clínica e ultra-sonografia, citados por $54 \%$ e $38 \%$ dos hospitais componentes de conglomerados; e os serviços de patologia clínica, radiologia, ultra-sonografia, hemoterapia, tomografia e hemodinâmica, terceirizados em mais de $50 \%$ dos hospitais especiais, segmento que apresentou maior terceirização de SADT.

A terceirização não se mostrou intensa para serviços médico-assistenciais, apresentando maior índice nos hospitais especiais. Entre eles, os mais citados, por segmento, foram: a anestesiologia, em oito hospitais da amostra; a cardiologia, em três grandes hospitais individuais; a anestesiologia e a hematologia, em, respectivamente, 25 e 18 dos hospitais componentes de conglomerados; e a cardiologia e traumato-ortopedia, respectivamente em 5 e 4 dos 10 hospitais especiais.

Observou-se pouca terceirização de serviços logísticos, principalmente nos grandes hospitais individuais. Nos hospitais da amostra, os 
serviços de contabilidade e de manutenção foram reportados como terceirizados parcial ou integralmente em, respectivamente, $42 \%$ e $41 \%$ dos casos.

\section{Discussão}

Considerados o tamanho de seu parque hospitalar, sua grande participação na produção de internações para o SUS e sua dispersão e interiorização no território nacional, os hospitais filantrópicos merecem atenção especial no que concerne à formulação de políticas públicas na área da Saúde. No entanto, o setor como um todo precisa ser compreendido em sua diversidade, com realidades diversas, exigindo, naturalmente, diferentes ações e tratamentos. Podem ser destacados como distintos: os hospitais da amostra, representando parte substantiva do parque filantrópico, na sua maioria com limitada complexidade assistencial e capacidade de gestão; os grandes hospitais individuais e os hospitais especiais, com alta complexidade assistencial e melhores condições gerenciais; e os conglomerados hospitalares, numa situação intermediária entre os achados da amostra e os grandes hospitais individuais.

Apreende-se a necessidade de desenvolvimento do setor. Inúmeras estruturas e instrumentos gerenciais básicos sob lógicas minimamente racionalizadoras e voltadas para a eficiência, encontram-se ausentes em boa parte dos hospitais. Não houve, entre os quesitos considerados, exigências mais avançadas e inovativas em termos gerenciais, salvo por exceção. Além disso, não se foi além do registro das estruturas e instrumentos de gestão, sem levar em conta a adequação dos mesmos à prática gerencial e ao desempenho organizacional. Considerando a importante prevalência de casos incipientes entre os hospitais da amostra, menores e menos complexos, depreende-se uma situação preocupante. Em tese, dados os riscos de sobrevivência, são esses que mais demandariam adequada incorporação e uso eficiente de instrumentos de gestão, exigindo assim medidas de ajustes, seja por parte dos agentes públicos, seja pelos próprios dirigentes dessas organizações.

A gestão de seus recursos humanos em organizações prestadoras de serviços ganha condição altamente estratégica para o alcance de bons resultados financeiros e, principalmente, relacionados à qualidade da atenção prestada. Dada a importância dos médicos para o efetivo funcionamento dos hospitais, a dominância de vínculos frágeis entre esses profissionais e suas organizações sugere a pertinência de estudos aprofundando o conhecimento sobre a dinâmica atual e as tendências desta força de trabalho.

É preciso explicar melhor as causas e as conseqüências da elevada rotatividade de pessoal observada, ainda que se assuma que comparações entre a rotatividade do setor saúde, e, mais especificamente, hospitalar, não devem ser feitas de forma automática, com a de outros setores da economia, dadas as especificidades do processo de trabalho. É possível que a enfermagem constitua-se na categoria profissional que mais contribui para a elevada rotatividade da mão-de-obra hospitalar, quer pela organização do trabalho (divisão técnica de tarefas etc.), quer pelas condições de trabalho (salários, carga horária etc.), quer pelo perfil do profissional (majoritariamente mulheres, em idade fértil, com filhos etc.) 17.

Ainda para o conjunto de hospitais, sobretudo os pequenos, de porte financeiro micro a médio, há a necessidade de melhor avaliação econômica. Mantidas as atuais condições, seriam esses hospitais sustentáveis no médio prazo? As exigências de investimento e desenvolvimento assistencial para boa parte deles gerará organizações sustentáveis? A análise dos balanços e demonstrativos de resultados, embora de grande valia para uma compreensão da realidade econômica do setor, não permite maiores conclusões em termos de tendências 18. Estas seriam forçosamente condicionadas por planos em curso, tanto de natureza mais setorial (discriminando as diferentes estratégias por tipo, localização e porte de hospitais, respeitados seus específicos mercados), quanto movimentos específicos internos a cada unidade, que devem ser identificados e estudados, na perspectiva da sua sustentabilidade.

Considerando o segmento de conglomerados hospitalares, o estudo não possibilitou análise aprofundada e específica quanto ao modelo de gestão neles praticado. Esse segmento deve ser investigado, especialmente na perspectiva de constituição de redes de colaboração entre hospitais como uma possível estratégia de desenvolvimento setorial, com vistas a garantir maior eficiência financeira, gerencial e assistencial 19

Há hospitais que apresentam condições gerenciais, em um ou em vários sistemas administrativos e assistenciais, que podem ser tomados como referência. Dentre outras estratégias de fortalecimento do setor, é possível incentivar-se o intercâmbio entre entidades e hospitais do próprio segmento. Experiência semelhante foi desenvolvida com sucesso no Ministério da Saúde mediante conformação de 
uma rede colaboradora entre hospitais. Essa estratégia visa a associar hospitais benchmarking em algum setor ou atividade a outros hospitais com perspectivas e condições para aprimoramento.

Identifica-se a necessidade de se investigar mais profundamente aspectos referentes à organização e qualidade clínico-assistencial desse parque hospitalar. Embora variáveis dessa dimensão constassem do questionário da pesquisa, não foram consideradas em função do baixo índice de respostas e limitada confiabilidade dos dados coletados. Entretanto, sublinha-se essa limitação, levando em conta a importância da gestão ou governança clínica no contexto da gerência de sistemas e organizações de saúde, vista hoje como um dos seus pontos críticos 10,20,21.

\section{Resumo}

Este artigo apresenta uma caracterização gerencial dos hospitais filantrópicos no Brasil, a partir de dados de um estudo nacional desenvolvido ao longo de 2001. Foram incluídas aleatoriamente 66 entidades prestadoras de serviços ao SUS com menos de 599 leitos, 26 entidades com pelo menos 599 leitos e dez não prestadoras de serviços ao SUS. As análises realizadas são descritivas, focalizando a classificação do nível de desenvolvimento gerencial dos hospitais e aspectos concernentes ao uso de instrumentos gerenciais específicos, recursos humanos, serviços técnicos e terceirização de serviços. Foram identificados distintos niveis gerenciais, merecendo destaque o fato de $83 \%$ dos hospitais prestadores de serviços ao SUS com menos de 599 leitos terem sido classificados como incipientes. Discutem-se implicações do quadro observado para as políticas de assistência hospitalar no país, considerando a importância desse parque hospitalar.

Hospitais; Hospitais Filantrópicos; Administração Hospitalar; Gerência
Os limitados dados assistenciais encontrados refletem a precariedade dos sistemas de informações desses hospitais. Atenção diferenciada deve ser destinada por parte dos formuladores de política pública para programas e incentivos que possibilitem a melhoria e, em alguns casos, a efetiva implementação deste instrumento indispensável para a gestão e avaliação das organizações.

Finalmente, identifica-se a necessidade de revisão e validação do sistema de classificação construído, incorporando dimensões e variáveis não consideradas e eliminando outras, para tornar o sistema mais sensível e de mais fácil aplicação de modo extensível ao conjunto de hospitais, sejam eles públicos ou privados, resguardadas as necessárias adaptações.

\section{Colaboradores}

Todos os autores tiveram participação ativa no desenho da pesquisa e na análise dos dados coletados. S. M. L. Lima, P. R. Barbosa e M. C. Portela foram responsáveis pela redação do artigo. Todos os autores participaram da revisão final do texto.

\section{Agradecimentos}

Este trabalho reporta resultados do Estudo sobre os Hospitais Filantrópicos no Brasil, uma ampla pesquisa nacional desenvolvida ao longo de 2001, pela Escola Nacional de Saúde Pública, Fundação Oswaldo Cruz, em colaboração e com financiamento do Banco Nacional de Desenvolvimento Econômico e Social, e parcerias do Ministério da Saúde, Caixa Econômica Federal e Confederação Nacional das Santas Casas de Misericórdia e Hospitais Filantrópicos. 


\section{Referências}

1. Brasil. Decreto no 4.327, de 8 de agosto de 2002. Aprova critérios para concessão do certificado de filantropia para os hospitais. Diário Oficial da República Federativa do Brasil 2002; 9 ago.

2. Warden GL, Griffith JR. Ensuring management excellence in the healthcare system. J Healthc Manag 2001; 46:228-37.

3. Zey-Ferrel M. Dimensions of organizations: environment, context, structure, process, and performance. Santa Monica: Goodyear Publishing Company; 1979.

4. Stanwick PA, Pleshko LP. Relationships of environmental characteristics, formalized planning, and organizational design to performance. International Journal of Organizational Analysis 1995; 3:175-97.

5. Institute of Medicine. Crossing the quality chasm. Washington DC: National Academy Press; 2001.

6. Field MJ, Lohr KN. Clinical practice guidelines: directions for a new program. Washington DC: National Academy Press; 1990. (Publication IOM 90-08).

7. Keating CB. A systems-based methodology for structural analysis of health care operations. J Manag Med 2000; 14:179-98.

8. Chang LC, Lin SW, Northcott DN. The NHS performance assessment framework: a "balanced scorecard” approach? J Manag Med 2003; 16:34558.

9. Griffith JR, Alexander JA. Measuring comparative hospital performance. J Healthc Manag 2002; 47:41-57.

10. Griffith JR. Championship management for healthcare organizations. J Healthc Manag 2000; 45:17-31.

11. Donabedian A. Explorations in quality assessment and monitoring: the definition of quality and approaches to its assessment. Ann Arbor: Health Administration Press; 1980.
12. Donabedian A. The quality of care: how can it be assessed? JAMA 1988; 260:1743-8.

13. Kazandjian VA, Matthes N, Wicker KG. Are performance indicators generic? The international experience of the Quality Indicator Project ${ }^{\circledR}$. J Eval Clin Pract 2003; 9:265-76.

14. Sloan FA, Conover CJ, Provenzale D. Hospital credentialing and quality of care. Soc Sci Med 2000; 50:77-88.

15. Shen YC. The effect of financial pressure on the quality of care in hospitals. J Health Econ 2003; 22:243-69.

16. Mintzberg H. Criando organizações eficazes - estrutura em cinco configurações. São Paulo: Editora Atlas; 1995.

17. Anselmi ML. A rotatividade dos trabalhadores de enfermagem nos hospitais de Ribeirão Preto [Tese de Doutorado]. Ribeirão Preto: Escola de Enfermagem de Ribeirão Preto, Universidade de São Paulo; 1993.

18. Barbosa PR, Portela MC, Ugá MAD, Vasconcellos MM, Lima SML, Gerschman S. Hospitais filantrópicos no Brasil. Rio de Janeiro: Banco Nacional de Desenvolvimento Econômico e Social; 2002. (Caderno Social 5).

19. Chan B, Feldman R, Manning WG. The effects of group size and group economic factors on collaboration: a study of the financial performance of rural hospitals in consortia. Health Serv Res 1999; 34 (1 Pt 1):9-31.

20. Mendes EV. Os sistemas de serviços de saúde: o que os gestores deveriam saber sobre essas organizações complexas. Fortaleza: Escola de Saúde Pública do Ceará; 2002.

21. Scally G, Donaldson LJ. Clinical governance and the drive for quality improvement in the new NHS in England. BMJ 1998; 317:61-5.

Recebido em 07/Ago/2003

Versão final reapresentada em 20/Abr/2004

Aprovado em 29/Abr/2004 